\title{
An overview of, Biomarkers in Carcinoma breast and, the relevance of ER/PR and HER-2/neu markers.
}

\author{
Agrawal, S N \\ (Department of Surgery,BKM Government medical college,jagdalpur.Bastar.C.G./Ravishankar niversity,India.)
}

\begin{abstract}
Breast cancer is a most common site specific cancer in women. It is the leading cause of death from cancer, exceeded only by, lung cancer. Traditionally they are classified by TNM classification consisting of Tumour, node and metastasis. They are then staged and also histopathology is taken into consideration. It has been seen that the same stage cancer behave differently to same treatment. The answer lies in its molecular biology. There are various biomarkers which have prognostic and predictive values. Their assessment helps in individualising the treatment of carcinoma breast. In this article an attempt is made to describe the various biomarkers and their influence. The basic workup for carcinoma breast consists of staging, histological classification and at least ER/PR and HER 2/new status. The proliferation marker Ki-67 provides important prognostic information particularly for the patients with small tumour and no axillary lymph node involvement.

Keywords: Carcinoma breast, immunohistochemistry, hormone receptors, ER/PR, Her-2/neu.
\end{abstract}

\section{Introduction}

Breast cancer is the most common site specific cancer in women and is the leading cause of death in women aged 20-59 years [1], [2]. Increased exposure to oestrogen is associated with an increased risk for developing breast cancer where as reducing exposure is thought to be protective. So the factors that increase the number of menstrual cycle such as early menarche, nulliparity and late menopause are associated with increased risk. Breast cancer is the most common malignant tumour in American women and is second only to lung cancers as a cause of cancer related mortality.

Breast cancer is a heterogeneous disease showing marked clinical and morphological diversities as well as variability in prognosis and response to different therapeutic modalities. The existing histological classification system for breast cancer is far from being accurate in predicting the prognosis or selecting the appropriate treatment of a given patient. [3].

Morphologically identical tumours can display divergent outcomes and response to therapy. This is attributed to molecular class difference that exists amongst histologically similar cancer types. [4]. The development of molecular analytical methods like immunohistochemistry (IHC), nucleic acid in situ hybridization, gene expression profiling etc. has helped in identification of ER/PR, HER-2/neu status and many other gene/ proteins for more reliable and predictive and/or prognostic indicators.[5].

The earliest development in this field was identification of ER/PR receptors by IHC. Florescence in situ hybridization (FISH) helped in identification of HER-2/neu gene (Human Epidermal Growth Factor Receptor). These biomarkers are biochemical substances that are over produced by the cancer cells or by host, in response to presence of cancer. Tumour markers may be produced by normal gene but are non specific. They may also be produced by the mutated gene that gives rise to uncontrolled signalling for initiation, development and progress of cancer. The cancer specific bio markers may be present in serum or tissue sample. ER/PR HER$2 /$ neu is regularly used for identification and planning of breast cancer treatment.

This paper addresses the diverse spectrum of various biomarkers and their significance in prediction, prognosis and treatment of breast cancer. Special emphasis is given to ER/PR and HER-2/neu biomarker.

\section{The burden}

Carcinoma breast is the most site specific cancer in women and is the leading cause of death from cancer in women aged 20-59 years [1], [2]. The American cancer society's estimate for breast cancer in United States for 2014 are;-

- About 232,670 new cases of invasive breast cancer will be diagnosed in women.

- About 62,570 new cases of carcinoma in situ (CIS) will be diagnosed.

- About 40,000 will die from breast cancer.

Brest cancer is the second leading cause of death in women exceeded only by lung cancer.

Breast cancer burden have well defined variation by geography, regional life style, and racial or ethnic background. [6]. In general both breast cancer incidence and morbidity are relatively lower among the female population of Asia and Africa, nations that have not developed, westernised reproduction and dietary pattern. In 
European and North American women and women from heavily industrialised or westernised countries have substantially higher breast cancer burden.

\section{The bio markers}

Traditionally the cancer staging of breast cancer is done by clinical examination of lump, Axillary lymph node status and FNAC or biopsy. However it has been seen that clinically same stage disease behaves differently to the same treatment and prognosis. The answer to this paradox lies in molecular behaviour of tissue. There are various bio markers which not only influence prognosis or outcome of treatment but also help in planning and identification of appropriate course of modalities.

The breast cancer bio markers can be defined as biochemical substances that are over produced by the cancer tissue or by host in response to presence of cancer. Tumour markers can be produced by normal gene but are non specific. They may also produce by mutated gene that gives rise to uncontrolled signalling for initiation, development and progression of cancer.

They are broadly divided into serum and tissue biomarkers.

3.1 Serum markers-

3.1.1. Carcino embryonic Antigen (CEA).

3.1.2. MUC -1 gene product.

3.1.3. New serum markers.

3.1.3.1. Urokinase plasminogen activator (UPA) \&Plasminogen activation inhibitor. (PAI- 1)

3.1.3.2.Lysophosphatidic Acid. (LPA) \&Lysophosphatidyl choline (LPC).

3.2. Tissue Markers-

3.1.3.3. RAK antigen of 25,42 , and $120 \mathrm{KDa}$.

3.2.1. Ki-67. (MIB-1)

3.2.2. Hormone receptors.

3.2.3. HER-2/neu. Human epidermal growth factor receptor-2.

3. 3. Other Tissue markers -

3.3.1. Oncogene

-EGFR (Epidermal Growth Factor Regulator).

-Mutation involving P 53, nm 23, and retinoblastoma

Gene (rb).

3.3.2. Apoptosis related gene-

P 53.

Bcl-2.

Bax-bcl-2 ratio.

3.3.3. Markers of Angiogenesis-

3.3.3.1. VEGF (Vascular Endothelial Growth factor).

3.3.3.2. BFGF (Basic Fibroblastic Growth Factor).

3.4. Miscellaneous markers-

3.4.1. Risk factor bio markers.

3.4.2. Exposure bio markers.

3.4.3. Cyclin and cyclin dependent Kinase.

3.4.3.1. Cyclin D 1 protein.

3.4.3.2. P 27, P 21, p 28, ERK 1/2.

3.4.4. Cathepsin-D.

3.4.5. Cyclo-oxygenase - 2. (COX-2).

\subsection{Serum markers}

3.1.1. Carcinoembryonic antigen- it was first described by Gold and Fredman as an oncofoetal antigen present in tumours of digestive tract. [7]. It is a 200-kda glycoprotein. The clinical use of serum CEA testing in breast cancer was pioneered by investigators at MD Anderson cancer centre. [8]. The early studies demonstrated that decreasing serum CEA values reflected response to chemotherapy with patients in metastatic disease and that increasing CEA reflected progressive disease.

3.1.2. MUC-1 gene product-the MUC gene located on chromosome 1q21-24, encodes a 160 to 230 kda glycoprotein. The extracellular portion of MUC-1 gene product is present in serum, and it can be measured by CA 15.3 dual-monoclonal immunometric assay and the CA 27.29 competitive inhibition immunoassay. 
Hayes and co workers were among the first to demonstrate the clinical utility of the CA 15.3 test for the serial monitoring of patients with metastatic breast cancer [9]. Recent evidences suggest that the CA 15-3 and CA 27.29 tests for the MUC-1 gene product are useful for the early detection of recurrence in patients with early stage breast cancer. The goal of early detection of recurrence is to initiate treatment that will result in increased survival duration and on improvement in quality of life [10].

3.1.3. New serum tumour markers. - New serum tumour markers are proposed by few analysts [11].

3.1.3.1. Urokinase plasminogen activator (UPA) and its major enzyme inhibitor- The plasminogen activation inhibitor (PAI-1). UPA and UPA-1 are produced by rumour tissue and has been established as independent indicator of prognosis. The UPA: UPA-1 complex was present in much higher quantities in plasma of patients with advanced breast cancer than in patients with localised breast cancer or healthy women [12].

3.1.3.2. Lysophosphatidic acid (LPA) and Lysophosphatidyl choline (LPC) both function as extracellular signals via membrane receptors to activate cell proliferation and function. They are elevated in $90 \%$ of patients with early stage breast cancer.

3.1.3.3. RAK antigens are proteins of 25,42 , and $120 \mathrm{Kda}$. In recent studies more than $95 \%$ of breast tumours have demonstrated to contain these proteins, whereas only $10 \%$ of normal breast tissues were positive for these proteins by immunohistochemical detection.

3.2. Tissue markers.

Interest in tissue markers as prognostic indicators has been stimulated by the success of adjuvant chemotherapy for early stage, operable breast cancer [13]. A number of biomarkers are now available to evaluate prognosis of breast cancer patient [14].but all are not validated in large clinical trials. The routine and most used marker are Ki-67, the hormone receptor markers (ER, PR), and HER-2/neu Oncogene. These markers along with size of tumour and axillary lymph node status allows oncologist to assess the patients prognosis.

3.2.1. Ki-67 (MIB) is a monoclonal antibody that identifies a nuclear antigen found in cells in proliferative phase of cell cycle i.e. G1, S, G2 and M phases. They are not found in cells in resting phase (Go) [15]. Thus its expression is used as broad measure of proliferation. Quantification is required with 500 to 1000 nuclei being counted [16]. Its expression are positively correlated with P53 over expression, high S phase fraction, aneuploidy, high mitotic index, and high histological grade in human breast cancer specimen and re negatively correlated with oestrogen receptor content.

3.2.2. Hormone receptors- hormones play a very important role in development and progression of breast cancer. Oestrogen, oestrogen metabolites and progesterone all influence carcinoma breast. The ER/PR status is routinely done in biopsy specimen.

It can be measured by using immunohistochemical methods. A concentration of $10 \mathrm{fmol} / \mathrm{mg}$ or more of cytosol protein are generally considered positive for clinical purposes.

Enzyme immunoassay or immunohistochemical methods of measuring ER, in tissue, depends upon colorimetric reactions or an scoring system based on visual estimates of number and intensity of stained cells. These include the $\mathrm{H}$ score, which is based on summation of percentage of cells of different intensities and the quick score system [17].

The ER antibody, clone 1 D5, in combination with heat induced epitope retrieval (HIER) method can give reproducible results in formalin fixed paraffin embedded (FFPE) tissues.

Its influence on clinical course:--

- $\quad$-Patients with hormone receptor positive tumour survive two to three times longer after the diagnosis of metastatic breast disease than a patient with hormone receptor negative tumour.

- $\quad$-In post menopausal women treatment with hormone replacement therapy (HRT) increases the risk of breast cancer by $26 \%$ as compared to placebo [18].

- The patients with ER positive status have prolonged disease free survival after primary treatment, longer overall survival and longer survival duration after recurrence as compare to ER negative patients. This advantage is independent of axillary nodal status.

Oestrogen receptor positive status is associated generally with-

- $\quad$ Low grade Histology.

- $\quad$ Favourable nuclear grade.

- Low s phase fraction.

- $\quad$ Low proliferative Index.

- $\quad$ Low thiamidine labelling index. [19].

Influence on therapy:-

- Patients with tumour negative status for both estrogens and progesterone are not suitable candidate for hormone therapy. 
- Tumour positive for both receptors have $50 \%$ more response rate for hormone therapy.

- Tumour negative for both receptors have response rate less than $10 \%$.

- Tumour positive for either of the receptors have intermediate response rate of around 33\%.

- They are both benefitted by adjuvant or palliative hormone therapy.

- Tumours without hormone receptors are more sensitive to chemotherapy than hormone sensitive tumours, with markedly higher rate of pathological complete response [20].

- Patients with node positive tumours, ER negative breast cancer, biweekly Doxorubicin, cyclophosphamide plus paclitaxel lowers the risk of recurrence and death by more than $50 \%$ in comparison with low dose Cyclophosphamide, Doxorubicin and Fluorouracil.

- In patients with hormone sensitive tumours Tamoxifen reduces the risk of recurrence and death by $30 \%$.

- The ER, PR status has got both predictive and prognostic value. The presence of hormone receptor is a powerful predictive factor for the likelihood of benefit from adjuvant hormonal therapy including aromatic inhibitors e.g. anastrozole, latrozole etc. and Tamoxifen [21]. [22].

As a prognostic factor, ER and/or PR positivity is associated with reduced mortality compared to women with ER and/or PR negative disease. [23]. [24]. [25].

\subsubsection{Human Epidermal Growth Factor receptor-2 (HER-2/neu)}

It is a member of type 1 tyrosine Kinase family also known as c-erb B-2 and neu. It is an oncoprotein and is over expressed in $20 \%$ of invasive primary breast cancers. A healthy breast cell has two copies of the HER-2 gene. Some kind of the breast cancer starts when the breast cell has more than 2 copies of that gene, and those copies start over producing HER-2 protein, which in turn send signal to breast tissue for rapid division and fast growth. This is NOT inherited from parents; the most likely cause is ageing, wear and tear etc. whether the environment pollutants play a part is not known.

The protein is tested by immunohistochemistry (IHC). This test measures the production of HER-2 protein by tumour. The result is marked as $0,1+, 2+, 3+$ etc. The test is considered positive if the results are $3+$ or above. This is also tested by Fluorescence in situ Hybridization (FISH).

This test uses fluorescent probes to look at the number of HER-2 gene copies in tumour cell. If these are more than two copies of HER-2 gene then the cancer is labelled positive for HER-2.

Its influence on disease process:-

i. This gene is over expressed and amplified in $60 \%$ cases of ductal carcinoma in situ (DICS) and 20 to $30 \%$ in infiltrating ductal carcinoma.

ii. In Paget's disease it is almost $100 \%$ over expressed and amplified.

iii. Its amplification/over expression is associated with poor prognosis in patients with carcinoma breast both with positive and negative lymph node. [26]

Its influence on therapy:-

i. It's over expression in carcinoma breast patients makes them more responsive to chemotherapy and hormone therapy.

II. They are benefitted by Anti-Her-2/neu monoclonal antibody Trastuzumab. [27].

In HER-2/neu over expressed breast cancer they show better response to Doxorubicin based chemotherapy. In a study conducted by the Cancer and Leukaemia group B (the CACGB 8541 study), the HER-2/neu over expression was associated with longer survival duration in patients with early stage breast cancer and positive lymph node, who are receiving increasing doses of Doxorubicin.

\subsection{Other tissue markers}

3.3.1. Oncogene. Epidermal Growth Factor Receptor (EGPR). It is a large Trans membrane, tyrosine Kinase, cell surface receptor that binds epidermal growth factor and transforming growth factor alpha (TGF- $\alpha$ ) . It's over expression could result in unrestrained cell proliferation. Loss of tumour suppressor genes may also play an important role in prognosis. Examples are mutations involving P53, nm 23, and retinoblastoma gene. Less extensively studied proto Oncogene are c-ras and c-myc.

3.3.2. Apoptosis related gene. Apoptosis is a programmed cell death. P 53 plays a major regulatory role in G 1 phase of cell cycle. It's over expression is associated with highly proliferative features i.e. poor nuclear grade, HER-2/neu over expression, and aneuploidy. And this is independent of age, nodal status and tumour size. [28].

It is not an independent prognostic indicator but it's over expression is clearly related to other prognostic indicator and adverse outcome. 
Bcl-2 protein is an important molecule to control apoptosis.bax is a death signal protein induced by genotoxic stress and growth factor deprivation. In breast cancer over expression of Bcl-2 and a decrease in bax: bcl-2 ratio correlates with:-

- High histological grade.

- Presence of axillary node metastasis.

- $\quad$ Reduced disease fee and survival rate.

3.3.3. Markers of Angiogenesis

Cancer cells create new blood supply to sustain their growth, and supply of nutrients, oxygen etc. Tumour releases two pro angiogenic factors which help in sustaining tumour growth.viz.. VEGF. Vascular endothelial growth factor and basic fibroblastic growth factor (BFGF)

P 53 gene regulates the expression of an angiogenesis inhibitor thrombospondin. Mutation of P 53 gene facilitates neo vascularisation due to lack of production of thrombospondin.

VEGF- expression and angiogenesis index have prognostic and predictive significance in carcinoma breast. Bevacizumab is a monoclonal antibody to VEGF. Its use is approved by UD FDA (US Food and Drug Administration) for the use in metastatic breast cancer in combination with Paclitaxel chemotherapy. [29].

3.4. Miscellaneous markers

3.4.1. Risk factor bio markers

They are associated with increased cancer risk. It includes

- Familial clustering and inherited germ line abnormality.

- Proliferative breast disease with atypia.

- Mammographic densities.

3.4.2. Exposure bio markers

Carcinogen exposure like DNA adducts. [30].

3.4.3. Cycline and Cycline dependent Kinase

3.4.3.1. Cyclin D1 protein -It is a cell protein that regulates the progression through G1 phase, the protein being over expressed in $50 \%$ of breast cancers. There is an association between this, presence of ER $\alpha$ and response to endocrine therapy. [31].

3.4.3.2. - P27 is a Cyclin dependent Kinase that is regulated by P53. Staining in presence of P53 reactivity can predict poorer survival in patients treated with adjuvant chemotherapy [32].

-P21 is a Cyclin dependent Kinase that is regulated by P53. Lack of P21stainingin presence of P53 reactivity can predict poorer survival in patients treated with adjuvant chemotherapy. [33]

-P38. A member of the mitogen activated protein (MAP) Kinase pathway. Its presence in active form in breast carcinoma relates to good endocrine response. [34].

-ERK $1 / 2$ protein, also a member of MAP family, which has a key regulatory role, in interaction between estrogens and growth factor cross talk. Detection of its phosporylated form, relates to endocrine resistance. [35]. 3.4.4. Cathepsin D -is suspected of facilitating invasion and metastasis of breast cancer. High levels are found in $1 / 3$ of Breast cancer patients, who have positive lymph nodes. It is secreted 52-KDa protein precursor (pro CD) has mitogenic activity and is proteolytic to basement membrane. Its value as independent prognostic marker is uncertain.

3.4.5. Cyclo-oxygenase-2- COX-2 derived metabolites may contribute to pre malignant hyper proliferation, transformation, maintenance of tumour viability, tumour growth, invasion and metastatic spread. [36].

\section{Conclusion}

Breast cancer is the most common cancer in American women after skin cancer. It is also second most common cause of death from cancer exceeded only by lung cancer.

In clinical work up the breast cancers are classified by TNM classification consisting of Tumour, Node and Metastasis. It is further staged and also histopathogy is taken into consideration. It has been seen that the cancer behaves differently with same stage and treatment strategy. The cause of this paradox lies in its molecular biology. For the breast cancer there are many prognostic and predictive factors.

A prognostic factor is defined as measurable variable that correlates with the natural history of the disease. [37]. The breast cancer prognostic factor are Tumour size, axillary lymph node status, lymphatic vascular invasion, patients age, histological grade, histological subtype (e.g. tubular, colloid, papillary etc.),response to neo adjuvant therapy, ER/PR status, HER-2/neu status etc.

A predictive factor is defined as the factors associated with response to given therapy. In breast cancer predictive factors are ER/PR status, HER-2/neu amplification and/or over expression. Additional predictive and prognostic factors are gene expression profiling, uPA/PAI-1 over expression, bone marrow micro metastasis, P53 gene analysis, Cathepsin-D level, micro vessel density etc. 
Serum tumour marker CEA, CA 27.29, HER-2/neu provides important information that is used to make treatment decisions for patients with metastatic breast cancer. ER/PR status is excellent predictor of response to hormonal therapy in early stage and advanced stage breast cancers. Over expression of HER-2/neu is associated with poor prognosis and those patients can be selected for Trastuzumab based therapy. As our understanding of breast cancer improves serum and tissue markers are going to play an important role in identification of patients for selection of most effective treatment for individual patients and for monitoring tumour response and patients status. In other words identification of biomarkers in serum and/or tissue will help in individualising the treatment of carcinoma breast as well as prediction of outcome and prognosis.

\section{References}

[1] Gunie VF: epidemiology of breast cancer, in Bland KI, Cope land EM III (ed): The breast comprehensive management of benign and malignant disease (Philadelphia: WB saunders 1998) p 339

[2] Jemal A, et al cancer statics 2008. Ca breast j clin 58:71, 2008.

[3] Cleator S, Ashworth A. Molecular profiling of breast cancer, Clinical implications. Br J cancer 200; 90: 1020-4.

[4] Bast RC jr, Ravdin P, Hayes DF, Bates S, Friksche H jr, Jessup JM et al. Update of recommendation for the use of tumour markers in breast and colorectal cancers. Clinical practice guidelines, of the American society, of clinical oncology. J clin oncol 2001; 19: 1865-78.

[5] Puzztai L, Mazounic, Anderson K, Wu Y, Symmans WF. Molecular classification of breast cancer, limitation and potential. Oncologist 2006; 11: 868-77.

[6] Ferlay J, et al: globocon 2002: cancer incidence, mortality and prevalence worldwide. Lyon, France: IARC press, 2004. IARC base no 4 , version 2.0 .

[7] Gold P, freedman SO, specific Carcinoembryonic antigen of the human digestive system. J exp med 1965: 122: 467-841.

[8] Mughal AW, Hortobagyi GN, Fritsche HA,Budzdar AU, Yap HY, Blumenschein GR. Serial plasma carcinogenic antigen measurement during treatment of metastatic breast cancer JAMA 1983: 249: 1881-1886.

[9] Hayes DF, zurawaski VR, Kuffe DW. Comparison of circulating CA15-3 and Carcinoembryonic antigen levels, in patients with breast cancer. J clin oncology 1986:4:1542-1550.

[10] Frische HA. Serum tumour markers for patient monitoring: a case oriented approach illustrated with Carcinoembryonic antigen. Clin chem. 1993:39: 2431-2434.

[11] Frische HA, Liu FJ, tumour markers at millennium. Proceedings of tumour markers conference (Santa Barba, CA 1999) Journal clinical ligand 2000:22: 320.

[12] Pedersen AN, Brunner N, Hoyer-Hansen G, et al.demonstration of the complex between Urokinase and its type-1 inhibitor in plasma from healthy donors and breast cancer patients. Clin chem. 1999:45: 1206-1213.

[13] Esteva FJ, Hortobagyi GN, Adjuvant systemic therapy for primary breast cancer. Surg clin North Am 1999; 79: 1075-1090

[14] Porter- Jordan k,Lippman Me. Overview of the biological markers of breast cancer. Haematol oncol clin North Am 1994; 8: 73-100.

[15] Tuccari G, et al. PCNA/Cyclin expression in breast carcinoma. Its relationship with Ki-67, ER, PgR immunostainings and clinico pathologic aspects. Pathologica 85; 47: 1993.

[16] Pinder SE, wencyk P, Sibbering DM, Bell JA, Eleston CW, Nicholson R, et al. Assessment of new proliferation markers MIB 1 in breast Carcinoma using image analysis: association with other prognostic factors and survival.Br. j. Cancer 1995; 71 : 146-9.

[17] Leake R, Barnes d, Pinder S, Ellis I, Anderson L, Anderson T, et al. Immunohistochemical detection of steroid receptors in breast cancer; a working protocol. J clin pathol 2000; 53: 634-5.

[18] Chlebowski RT et al. Influence of oestrogen plus progesterone on breast cancer and mammography in healthy post menopausal women. The womens health initiative randomised trials. JAMA 289;3243: 2003.

[19] Donegan WL. Tumour related prognostic factors for breast cancer. CA Cancer J Clin 1997; 47: 28-51.

[20] Donald A,Berry, PhD et al. Oestrogen receptor status and outcome of modern chemotherapy for patients with node positive breast cancer . JAMA 2006; 295 (147): 1658-1669.

[21] Paik S, Shak S, Tang G, et al. A multigene assay to predict recurrence of Tamoxifen treated node negative breast cancer. N Engl J med 2004; 351 (27): 2817-26.

[22] Von't, Veer LL, Dai H, Van de Vijvir MJ, et al. Gene expression profiling predicts clinical outcome of breast cancer. Nature 2002; 415 (6871): 530-6.

[23] Fisher B, Jeong JH, Bryant J, et al. Treatment of lymph node negative, oestrogen receptor positive breast cancer. Long term findings from National Surgical Adjuvant Breast and bowel project randomised clinical trials. Lancet 2004; 364 (9437): 858-68.

[24] Winer EP, Hudis C, Burestein HJ, et al. American society of clinical oncology technology assessment on use of aromatase inhibitors as adjuvant therapy for post menopausal women with hormone receptor positive breast cancer; status report 2004.J clin oncol,2005; 23(3): 619-29.

[25] Tamoxifen for early breast cancer; an over view of the randomised trials. Early breast cancer trialists collaborative group Lancet 1998; 351(9114): 1451-67.

[26] Esteva- Lorenzo FJ, Sastry L, King CR. the erb B-2 gene, from research to application. In Dickson RB, Salomon DS, eds hormones and growth in development and neoplasia New York, John Wiley \& sons; 1998.

[27] Ross JS, Fletcher JA. The HER-2/neu oncogene.prognostic factor, predictive factor and target for therapy. Semin Cancer Biol 1999; 9: $125-138$.

[28] Borresen AL,Anderson TI, Eyfjord JE, et al. P53 mutation and breast cancer prognosis. Particularly poor survival rates for cases with mutations in the zinc- binding domains. Gene's chromosomes cancer 1995; 14: 71-75.

[29] Millar K et al. Paclitaxel plus Bevacizumab versus paclitaxel alone for metastatic breast cancer. N Engl j med 357; $2666: 2007$.

[30] Dhillon DA, Molecular markers in diagnosis and staging of breast cancer. Semin radial oncol; 12:329: 2002.

[31] Umekita Y, Ohi Y, Sgara Y, Yoshida H. Over expression of Cyclin D1 predicts for poor prognosis on oestrogen receptor negative breast cancer patients, int J cancer 2002; 98: 415-8.

[32] Areaga CL. CDK inhibitor P27 Kip1 and hormone dependence in breast cancer. Clin Cancer Res 2004; 10 suppl: $3685-715$.

[33] Thor AD, Liu S, Moore DH II, Shi Q, Edgerton SM. P21 WAF 1/CIP1 expression in breast cancers, associations with P53 and outcome. Breast Cancer Res Treat 2000; 61: 33-43.

[34] Gee JMW, Robertson JFR, Ellis IO, Nicholson RI. Impact of activation of MAP Kinase family members, on endocrine response and survival in breast cancer. Eur j Cancer 2000; 198: 450-7. 